\title{
Research Article \\ Effect of Film-Forming Polymers on Release of Naftifine Hydrochloride from Nail Lacquers
}

\author{
Indrè Šveikauskaitè and Vitalis Briedis \\ Department of Clinical Pharmacy, Lithuanian University of Health Sciences, Sukileliu Pr. 13, 50161 Kaunas, Lithuania \\ Correspondence should be addressed to Indrè Šveikauskaitė; indre.sveikauskaite@lsmuni.lt
}

Received 4 November 2016; Revised 11 January 2017; Accepted 23 January 2017; Published 16 February 2017

Academic Editor: Jaume Garcia-Amorós

Copyright (c) 2017 Indrè Šveikauskaitè and Vitalis Briedis. This is an open access article distributed under the Creative Commons Attribution License, which permits unrestricted use, distribution, and reproduction in any medium, provided the original work is properly cited.

\begin{abstract}
The successful topical therapy of onychomycosis depends on effective drug release and penetration into nail, which can be achieved by using an adequately developed delivery system. This study evaluated and compared effect of film-forming polymers Eudragit RL100, Eudragit RS100, and ethyl cellulose on naftifine hydrochloride release from experimental nail lacquer formulations. Quality of formulations was evaluated by determining drying time and water resistance. Interactions between active pharmaceutical ingredient and excipients were investigated using microcalorimetry and FT-IR. Optimization of nail lacquer formulations was performed by naftifine hydrochloride release testing. Release of naftifine hydrochloride increased with increasing concentration of Eudragit RL100. Plasticizer triacetin affected the release of naftifine hydrochloride, when Eudragit RS100 polymer was used. Ethyl cellulose polymer was determined to be not applicable for naftifine hydrochloride nail lacquer formulations. Two compositions of nail lacquers were optimized and could be used in further development of transungual delivery systems.
\end{abstract}

\section{Introduction}

Onychomycosis is a fungal infection of nails that causes discoloration, thickening, and separation from the nail bed. It affects up to $14 \%$ of the population and is responsible for almost $50 \%$ of nails diseases [1]. Oral antifungal therapy remains the most often used for onychomycosis treatment; however, it has significant disadvantages: long systematic treatment can cause serious side effects, liver damage, and only tiny drug fraction could reach a target because of limited blood circulation [2]. Therefore, topical therapy is often considered as reasonable alternative. Topical delivery systems offer many benefits in dermatology; however, gel, cream, or liquid formulations are not adequate for the transungual delivery since they are easily removed by washing or rubbing. This phenomenon at the site of application accounts for their inefficacy [3-6].

Few days lasting drug delivery is considered as essential requirement for pharmaceutical formulations applied topically on the nail $[7,8]$. Film-forming systems for transungual drug delivery are used in clinical practice, but their ability to deliver therapeutic doses of active substances remains critical for efficient onychomycosis treatment $[7,9,10]$.

Mechanical characteristics and water resistance make nail lacquers a promising alternative for onychomycosis topical treatment. Lacquer film is formed during solvents evaporation after lacquer application. Films formed by nail lacquers have to establish attachment to the nail surface which is a necessary prerequisite of efficient drug delivery [11]. The increase of drug concentration depends on the volatile fraction in the nail lacquer [12]. This system is considered to be efficient for onychomycosis therapy as a film creates a reservoir of the drug substance in the infected nail plate. This leads to achieving continuous penetration of drug substance, required for an effective treatment [13-15].

Reservoir properties and release characteristics are affected by nature of the polymer used for film formation. Polymeric film can form an external reservoir, control a release of drug substance, and (or) limit the supply of drug [16]. Differences in film-forming system technology can affect film affinity to keratin and nail permeation [17]. Drug release from film-forming systems is affected by several factors: 
TABLE 1: Composition of $1 \%$ naftifine hydrochloride nail lacquers.

\begin{tabular}{lcccccccccccccccccccc}
\hline & N1 & N2 & N3 & N4 & N5 & N6 & N7 & N8 & N9 & N10 & N11 & N12 & N13 & N14 & N15 & N16 & N17 & N18 & N19 & N20 \\
\hline API & 1 & 1 & 1 & 1 & 1 & 1 & 1 & 1 & 1 & 1 & 1 & 1 & 1 & 1 & 1 & 1 & 1 & 1 & 1 & 1 \\
$\begin{array}{l}\text { Solvents system } \\
(1: 1: 1)\end{array}$ & 83.4 & 84 & 81.5 & 81.5 & 84 & 81.2 & 85 & 79 & 85 & 81.2 & 84 & 81.5 & 83.4 & 84 & 81.5 & 81.5 & 84 & 81.2 & 85 & 79 \\
Triacetin & 2.8 & 0 & 5 & 2.5 & 5 & 3.9 & 2 & 5 & 2 & 3.9 & 5 & 2.5 & 2.8 & 0 & 5 & 2.5 & 5 & 3.9 & 2 & 5 \\
Eudragit RL100 & 12.8 & 15 & 12.5 & 15 & 10 & 13.9 & 12 & 15 & - & - & - & - & - & - & - & - & - & - & - & - \\
Eudragit RS100 & - & - & - & - & - & - & - & - & - & - & - & - & 12.8 & 15 & 12.5 & 15 & 10 & 13.9 & 12 & 15 \\
Ethyl cellulose & - & - & - & - & - & - & - & - & 12 & 13.9 & 10 & 15 & - & - & - & - & - & - & - & - \\
\hline
\end{tabular}

active substance physicochemical properties, type and concentration of a polymer and plasticizer, and incorporation of other excipients $[18,19]$.

The aim of this study was to evaluate the effect of filmforming polymers on quality characteristics of nail lacquers and naftifine hydrochloride release. Optimization of lacquer formulations was performed referring to naftifine hydrochloride release data.

\section{Materials and Methods}

2.1. Materials. Naftifine hydrochloride was purchased from Chemical Point (Deisenhofen, Germany). Ethanol 96\% (AB Stumbras, Lithuania), butyl acetate, and ethyl acetate were obtained from Sigma-Aldrich Chemie GmbH (Steinheim, Germany) and used as solvents system. Triacetin which was used as plasticizer was kindly supplied by Lanxess (Germany). Film-forming polymers Eudragit RL100 and Eudragit RS100 were kindly gifted by Evonik Industries AG (Essen, Germany), while ethyl cellulose Ethocel Standard 20 Premium was obtained from Colorcon (UK).

\subsection{Methods}

2.2.1. Preparation of Nail Lacquers. The experimental nail lacquers were formulated by dissolving appropriate amount of naftifine hydrochloride in a base lacquer, containing ethanol (96\%), butyl acetate, ethyl acetate, triacetin, and one of the film-forming polymers: Eudragit RL100, Eudragit RS100, or ethyl cellulose. Experimental compositions of $1 \%$ naftifine hydrochloride nail lacquers are presented in Table 1.

Naftifine hydrochloride was mixed with solvent system using magnetic stirrer at $1000 \mathrm{rpm}$ until the drug substance was completely dissolved. Polymer was added and mixed until a clear solution was obtained. The plasticizer was added on the final step of the lacquer formulation.

2.2.2. Physical Characterization of Nail Lacquers. Quality of nail lacquers was evaluated by measuring drying time, water resistance, compatibility of drug with excipients, and ratio of released and absorbed heat.

Drying time was determined by applying a liquid film of experimental sample on a glass plate and time till obtaining dry-to-touch condition at room temperature $\left(20 \pm 2^{\circ} \mathrm{C}\right)$ was measured. A smoothness of flow was determined by
TABLE 2: Conditions and duration of the microcalorimetric testing.

\begin{tabular}{lcc}
\hline Temperature ranges & Change speed & Duration \\
\hline $20-100^{\circ} \mathrm{C}$ & $1 \mathrm{~K} / \mathrm{min}$ & $4800 \mathrm{~s}$ \\
$100-200^{\circ} \mathrm{C}$ & $0.5 \mathrm{~K} / \mathrm{min}$ & $12000 \mathrm{~s}$ \\
$200-20^{\circ} \mathrm{C}$ & $1.2 \mathrm{~K} / \mathrm{min}$ & $9000 \mathrm{~s}$ \\
\hline
\end{tabular}

spreading the sample on a glass slide and rising it vertically [20].

The water resistance test was conducted by applying a nail lacquer onto a glass slide, allowing it to dry, then immersing a glass slide with the lacquer film in water for 7 days [21]. Changes in film discoloration, turbidity, and resistance to water were evaluated every 24 hours. Structural changes of films were visualized by means of inverted microscope Olympus IX71 combined with LCAchNYOXPH lens.

A ratio of released and adsorbed heat of developed formulations was determined using microSC microcalorimeter (Setaram Instrumentation, France). Experiment was carried out in two phases: heating and cooling of experimental formulations. Conditions of the experiment are presented in Table 2.

FT-IR spectra were used to evaluate the possible existing molecular interactions between drug and film-forming polymer. Drug-excipients compatibility studies were performed by scanning from 4000 to $400 \mathrm{~cm}^{-1}$ in a FT-IR Spectrum Two spectrophotometer (PerkinElmer, USA). Peak matching was done to detect any possible interactions of components.

2.2.3. In Vitro Naftifine Hydrochloride Release Testing. Nafti fine hydrochloride release experiments were carried out at $32^{\circ} \mathrm{C}$ measuring drug quantity diffusing through $1 \mathrm{~cm}^{2}$ cuprophan dialysis membranes (MWCO - $10000 \mathrm{Da})$. Diffusion membrane was mounted on the diffusion cell and $50 \mu \mathrm{L}$ of lacquer experimental formulation was applied uniformly on the surface of the membrane and left for 4 hours till complete lacquer drying and film formation. Drying time of 4 hours was experimentally proven to be sufficient to achieve dry lacquer film under ambient conditions by determining weight loss kinetics. Dry film was protected from external factors by aluminum foil cover. Naftifine hydrochloride solubility in water at $32^{\circ} \mathrm{C}$ was determined to be $950.1 \mu \mathrm{g} / \mathrm{mL}$ and water $(15 \mathrm{~mL})$ was used as acceptor phase for establishing sink conditions. At predefined time points, $2 \mathrm{~mL}$ of acceptor 
TABLE 3: Quantitative ranges of components in nail lacquer formulations.

\begin{tabular}{lcc}
\hline Component & Function & Amount $(\%)$ \\
\hline Ethanol (96\%) : ethyl acetate : butyl acetate & Solvents system & $79-85 \%$ \\
Eudragit RL100/Eudragit RS100/ethyl cellulose & Film-forming polymer & $10-15 \%$ \\
Triacetin & Plasticizer & $0-5 \%$ \\
\hline
\end{tabular}

medium was withdrawn and the same amount of a fresh medium was added to maintain a constant volume of acceptor liquid. The amount of released drug was determined by UV spectrophotometry at $294 \mathrm{~nm}$ wavelength in triplicate. The released drug amount was calculated and results were denoted as the means \pm SD.

2.2.4. Optimization of Compositions. The compositions of nail lacquers for optimization were generated by applying D-optimal design model according to quantitative ranges of components (Table 3). Eight different compositions were generated for each film-forming polymer. Optimization of experimental nail lacquers formulations was performed by setting in vitro release results as the critical criteria.

2.2.5. Statistical Analysis. Nail lacquers optimization was performed using experiment planning software DesignExpert 6.0.8, D-optimal design model. Statistical analysis of experimental data was performed using SPSS software (version 19.0) and Microsoft Office Excel 2015. Spearman's rank coefficient was used for correlation analysis. Statistically significant difference was determined when value of $P<0.05$.

\section{Results and Discussion}

Suitability of hydrophobic polymers for film formation from experimental formulations of nail lacquers and transungual delivery of naftifine hydrochloride was evaluated. The solubility of the film-forming polymer defines whether dispersion or solution of polymer will be applied for film formation, which in turn affects mechanical properties and appearance of formed film [7, 11] as well as drug release rates [22]. As the solvents evaporate the polymer chains come into closer contact in solutions resulting in formation of a smooth and water resistant film $[7,16]$. The choice of the filmforming polymers was made considering their solubility and the characteristics of the formed solutions. Eudragit RL100 and Eudragit RS100 polymers are freely soluble in the mixtures of organic solvents. These polymers formed clear, low viscosity, and runny solutions. Clear and homogenous film was produced by controlling polymer and plasticizer concentrations in nail lacquer. The characteristics of polymeric films formed by experimental nail lacquer formulations are presented in Table 4 . The viscosity of solutions increased as the concentration of the polymer was increased [11]. The increase of polymer concentration resulted in longer duration of film formation, but it had effect on dried lacquer film characteristics. Film drying time was also affected by addition of plasticizer. The drying time for the formulations containing $0-2 \%$ plasticizer was less than 60 seconds, and formulations with $5 \%$ plasticizer amount were drying in 3-4 minutes.

Published results have demonstrated the effect of the viscosity of the film-forming polymer solution on the smoothness of the formed film [11]. The viscosity of ethyl cellulose solution was in the range from 18 to $22 \mathrm{mPas}$ when concentration of the film-forming component ranged from 10 to $15 \%$, respectively. Ethyl cellulose showed poor solubility in the mixture of organic solvents. It formed clear, opalescent, viscous solutions. When the concentration of ethyl cellulose was higher than $12.5 \%$, precipitates containing white film have been formed. Addition of triacetin plasticizer to ethyl cellulose solution resulted in shorter drying times of the films. Nevertheless, the drying time of ethyl cellulose films ranged from 8 to 10 minutes, and the formed films remained sticky.

Presence of plasticizer in film-forming solution results in a stronger and flexible film [23]. Results of film testing demonstrated the ability of plasticizer to affect mechanical properties of the film and its increased resistance to water. Plasticization reduces the glass transition temperature of the polymers and provides film with higher flexibility, strength, and resistance $[7,11,23]$. Results of water resistance testing showed that film resistance depended on the amount of filmformer, plasticizer, and their ratio. Maximum stability and resistance to water were determined when lacquer formulations contained $13.9 \%$ Eudragit RL100 polymer and 3.9\% triacetin. Film color change to yellowish was determined on the 5th day of testing. When formulations contained no plasticizer, they were washed away in 24 hours. Films, produced from $15 \%$ ethyl cellulose and $2.5 \%$ plasticizer containing formulations, were washed away in 48 hours. The wash-out times were confirmed by microscopic evaluation of film surface.

Chemical interactions of film-forming polymers and plasticizer can result in the changes of film properties. Drug interactions with film-forming system can cause reduced release of the drug from the produced film. Possible interactions of the nail lacquer components were evaluated by microcalorimetry and FT-IR spectroscopy. Nail lacquer formulations containing Eudragit RL100 and Eudragit RS100 showed no change in absorbed thermal energy when compared with naftifine hydrochloride free formulations (Figure 1(a)). New peaks were observed in all thermographs of ethyl cellulose polymer films containing naftifine hydrochloride when temperature was raised to $190^{\circ} \mathrm{C}$. Changes of polymer concentration had no effect on thermal behavior of tested films. FT-IR analysis revealed no chemical interaction between naftifine hydrochloride and film-forming polymers 


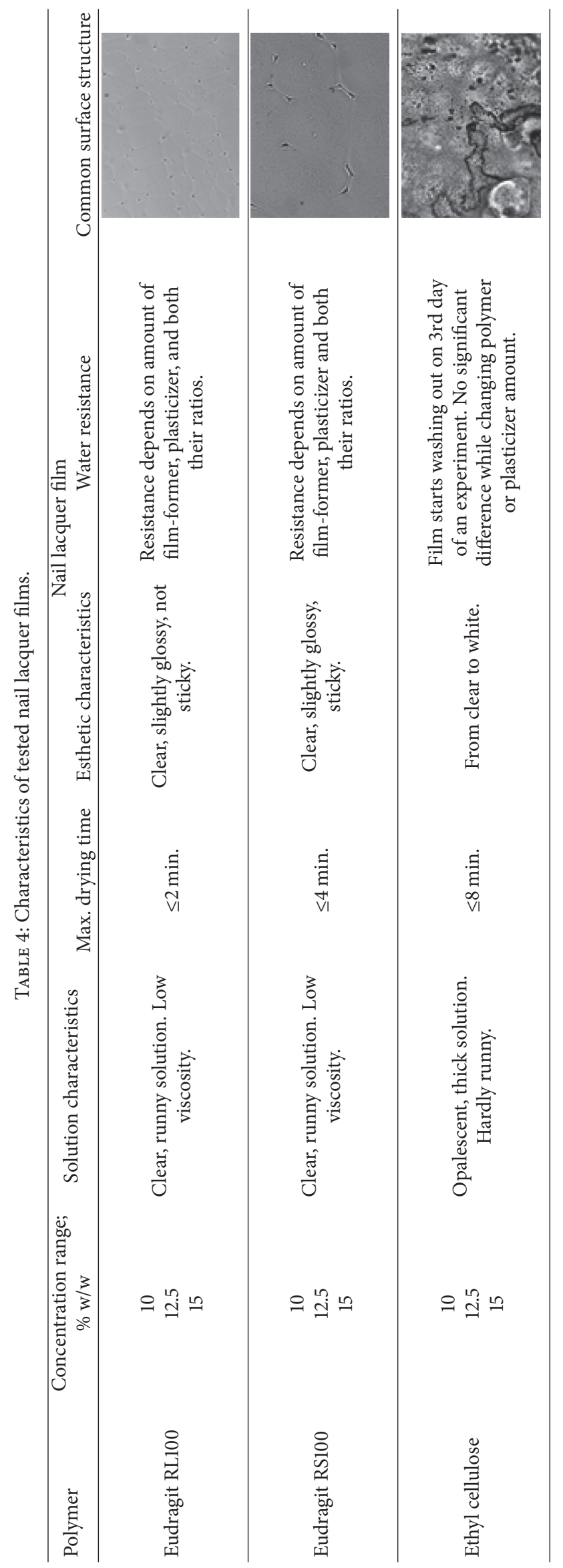




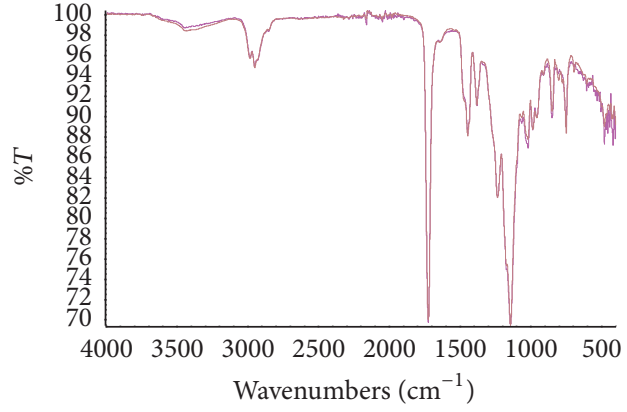

(a)

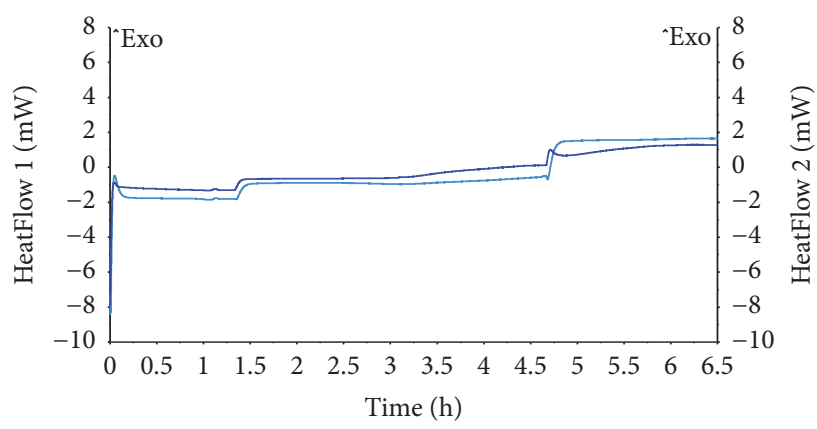

(b)

FIgURE 1: (a) FT-IR spectra of formulation N6. Red, API-free formulation; pink, naftifine hydrochloride nail lacquer. (b) Microcalorimetric thermograph of formulation N6. Dark blue, API-free formulation; light blue, naftifine hydrochloride nail lacquer. Duration of testing is approximately $6.5 \mathrm{~h}$.

TABLE 5: Naftifine hydrochloride release, polymer Eudragit RL100.

\begin{tabular}{|c|c|c|c|c|c|c|c|c|}
\hline \multirow{2}{*}{ (h) } & \multicolumn{8}{|c|}{ Formulation number } \\
\hline & N1 & $\mathrm{N} 2$ & N3 & $\mathrm{N} 4$ & N5 & N6 & N7 & N8 \\
\hline 0.25 & $27 \pm 1.2$ & $37.5 \pm 0.5$ & $33.5 \pm 0.92$ & $26.9 \pm 1.23$ & $26 \pm 0.8$ & $35 \pm 0.79$ & $39.7 \pm 1.12$ & $42.7 \pm 0.81$ \\
\hline 0.5 & $39.7 \pm 1.1$ & $47.2 \pm 0.6$ & $45.2 \pm 0.71$ & $35.25 \pm 1.31$ & $31 \pm 0.71$ & $47.2 \pm 0.91$ & $45.3 \pm 1.31$ & $52.4 \pm 0.93$ \\
\hline 1 & $49.1 \pm 1$ & $62.3 \pm 0.51$ & $53.8 \pm 0.82$ & $49.6 \pm 1.4$ & $45 \pm 0.9$ & $58.2 \pm 0.8$ & $57.8 \pm 1.24$ & $69.3 \pm 0.72$ \\
\hline 2 & $55.3 \pm 1.3$ & $70.8 \pm 0.82$ & $56.9 \pm 0.95$ & $62.2 \pm 1.2$ & $49.4 \pm 0.85$ & $67 \pm 1.13$ & $65.4 \pm 1.13$ & $78 \pm 0.85$ \\
\hline 3 & $58.7 \pm 0.9$ & $75.3 \pm 0.73$ & $63.2 \pm 0.75$ & $72 \pm 1.15$ & $52.3 \pm 0.62$ & $67 \pm 0.42$ & $69.9 \pm 1$ & $86 \pm 0.67$ \\
\hline 4 & $63.7 \pm 1$ & $77.9 \pm 0.6$ & $68.5 \pm 0.8$ & $74.4 \pm 1.2$ & $58.2 \pm 0.75$ & $68 \pm 0.93$ & $70 \pm 1.32$ & $94 \pm 0.97$ \\
\hline 5 & $66.7 \pm 1.2$ & $84.1 \pm 0.7$ & $73.1 \pm 0.72$ & $77 \pm 1.25$ & $60.2 \pm 0.82$ & $73 \pm 1.21$ & $72 \pm 1.25$ & $98 \pm 0.82$ \\
\hline 6 & $70.5 \pm 1.3$ & $90 \pm 0.62$ & $77.8 \pm 0.61$ & $80.9 \pm 1.5$ & $66 \pm 0.91$ & $77 \pm 1.32$ & $72.5 \pm 1.31$ & $98.5 \pm 0.86$ \\
\hline
\end{tabular}

(Figure 1(b)). Microcalorimetry revealed possible naftifine hydrochloride and ethyl cellulose can have internal interactions when the temperature was increased. These results suggest unsuitability of ethyl cellulose use as a film-forming polymer for naftifine hydrochloride delivery.

Published results confirm that the nature of chosen filmforming polymer can affect mechanical, cosmetic properties of dried film, and drug release kinetics from formed system $[7,21,22]$. In vitro release studies demonstrated significant differences between tested polymeric films. Statistically significant correlation was determined between increasing Eudragit RL100 concentration and increased amount of released naftifine hydrochloride (Table 5). Hydrophobic polymers can produce a "burst" release of the active substance and have ability to form a drug reservoir in biological membrane [16]. Highest release of drug substance $(98.5 \%$ in 6 hours) was achieved from nail lacquer film containing 15.0\% Eudragit RL100. In this case, presence of plasticizer had no effect on naftifine hydrochloride release rate, thus indicating it had no effect on the drug-polymer interaction in the formed polymeric film. However, plasticizer enhanced release of drug when Eudragit RS100 was used. Statistically significant correlation $(P<0.05)$ was established between increased polymer-plasticizer ratio and increased release rate of naftifine hydrochloride.

Conversely, the release of naftifine hydrochloride increased when concentration of Eudragit RS100 in nail lacquer was decreased (Table 6). This effect can be related to structural differences of Eudragit RL100 and Eudragit RS100 as higher number of quaternary ammonium substitution is present in Eudragit RL100 if compared to Eudragit RS100.

Possibility of using ethyl cellulose for film formation has been demonstrated with fluconazole [24]. Films produced from $10-11 \%$ ethyl cellulose containing formulations showed more than $90 \%$ release of fluconazole in less than 10 hours. Film formation is influenced by used solvent system which should efficiently dissolve the film-forming components and drug substance. Ethyl cellulose formulations delayed the release of naftifine hydrochloride as only $40 \%$ of the drug was released in $72 \mathrm{~h}$. This result should be considered acceptable, but the film wash away occurred in $72 \mathrm{~h}$.

Considering naftifine hydrochloride release in vitro results, formulations with Eudragit RL100 and Eudragit RS100 were chosen for further optimization studies. Optimization was performed by applying desirability function. Desirability of in vitro release parameter ranged between 0 and 1 , with values verging towards 1 considered the most desired. Optimal compositions of nail lacquers are presented in Figure 2.

Predicted in vitro release results were checked by performing in vitro release testing (Table 7). Optimized formulations were tested by evaluating drying time, water resistance, drug-excipients compatibility, and ratio of released and absorbed heat. 
TABLE 6: Naftifine hydrochloride release, polymer Eudragit RS100.

\begin{tabular}{|c|c|c|c|c|c|c|c|c|}
\hline \multirow{2}{*}{ (h) } & \multicolumn{8}{|c|}{ Formulation number } \\
\hline & N13 & N14 & N15 & N16 & N17 & N18 & N19 & N20 \\
\hline 0.25 & $12.6 \pm 1.22$ & $22 \pm 0.82$ & $16 \pm 0.87$ & $16 \pm 0.75$ & $25 \pm 0.87$ & $19 \pm 0.97$ & $21 \pm 1.25$ & $24 \pm 0.67$ \\
\hline 0.5 & $23.9 \pm 1.11$ & $23 \pm 0.91$ & $24 \pm 0.96$ & $16.4 \pm 0.84$ & $32 \pm 0.97$ & $21 \pm 0.91$ & $26 \pm 1.12$ & $25 \pm 0.87$ \\
\hline 1 & $43.8 \pm 1.2$ & $24.5 \pm 0.71$ & $32 \pm 0.78$ & $20 \pm 0.87$ & $42 \pm 0.68$ & $28 \pm 0.84$ & $35.9 \pm 1.14$ & $33.1 \pm 0.91$ \\
\hline 2 & $52.8 \pm 1.34$ & $27 \pm 0.62$ & $46.4 \pm 0.89$ & $26 \pm 0.75$ & $46 \pm 0.78$ & $33 \pm 0.79$ & $41.3 \pm 1.08$ & $43.8 \pm 0.84$ \\
\hline 3 & $62.8 \pm 1.22$ & $30.1 \pm 0.87$ & $56.2 \pm 0.87$ & $31.5 \pm 0.94$ & $52 \pm 0.89$ & $40 \pm 0.84$ & $48.7 \pm 1.89$ & $54.3 \pm 0.67$ \\
\hline 4 & $71.5 \pm 1.25$ & $32 \pm 0.89$ & $61.5 \pm 0.86$ & $34.3 \pm 0.78$ & $62 \pm 0.95$ & $61 \pm 0.94$ & $59 \pm 1.7$ & $71.6 \pm 0.87$ \\
\hline 5 & $76.1 \pm 1.31$ & $33.5 \pm 0.75$ & $66.3 \pm 0.87$ & $40.1 \pm 0.68$ & $63 \pm 0.78$ & $62 \pm 0.74$ & $62 \pm 1.54$ & $72.8 \pm 0.57$ \\
\hline 6 & $78 \pm 1.15$ & $36.5 \pm 0.96$ & $69.35 \pm 0.64$ & $51 \pm 0.79$ & $66 \pm 0.87$ & $64 \pm 0.85$ & $67 \pm 1.34$ & $73 \pm 0.97$ \\
\hline
\end{tabular}

TABLE 7: Characteristics of optimized nail lacquer formulations.

\begin{tabular}{|c|c|c|c|c|c|}
\hline \multirow{2}{*}{ Optimized formulation } & \multicolumn{3}{|c|}{ Components of formulation } & \multirow{2}{*}{ Designed in vitro release value } & \multirow{2}{*}{ Determined in vitro release value } \\
\hline & Solvents & Polymer & Triacetin & & \\
\hline OPT-1 & $79 \%$ & $15 \%$ & $5 \%$ & $96.19 \%$ & $98 \%$ \\
\hline OPT-2 & $83.4 \%$ & $10.6 \%$ & $5 \%$ & $81.38 \%$ & $80.5 \%$ \\
\hline
\end{tabular}

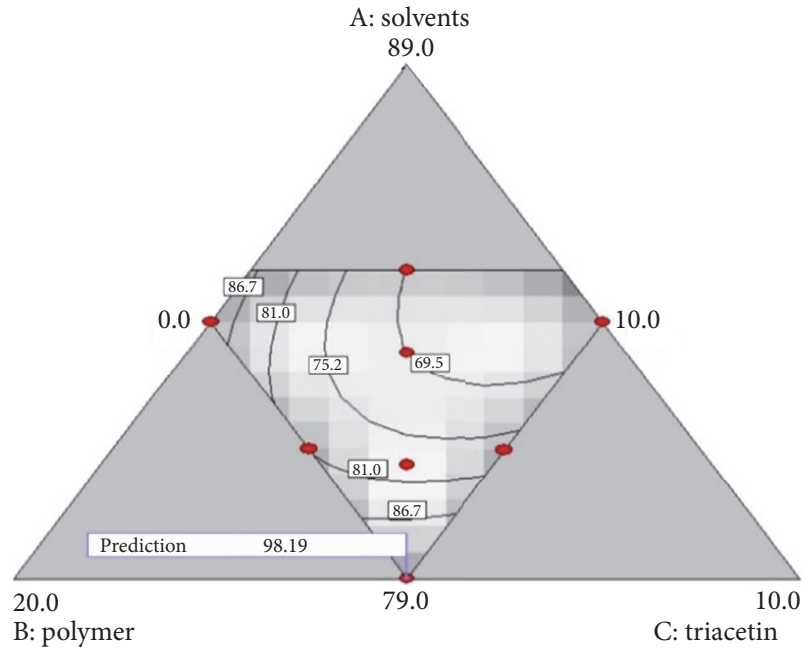

(a)

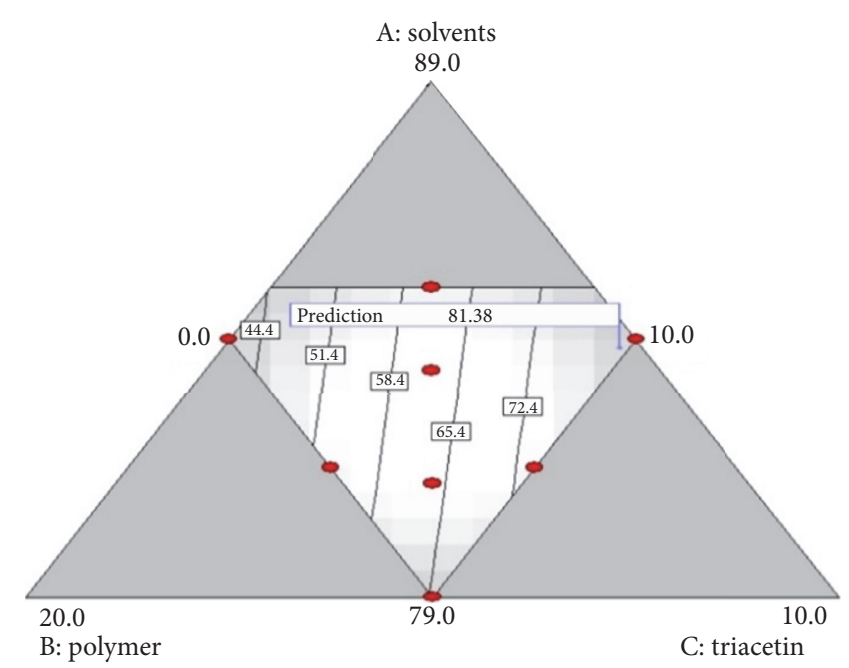

(b)

FIGURE 2: Areas of optimal composition of nail lacquers with highest desirability parameter: (a) OPT-1 formulation, Eudragit RL100 polymer; (b) OPT-2 formulation, Eudragit RS100 polymer.

\section{Conclusions}

Antifungal formulations for transungual drug delivery represent complex systems, influenced by solubility characteristics of used polymers, plasticizers, and physicochemical characteristics of the drug. System of evaporating solvents used in formulation of lacquers should ensure dissolution of formulation components and formation of homogeneous product before and after film drying. Application of adequate quality testing methods provides reliable information on possible interactions of lacquer system components and for biopharmaceutical characterization of the drug containing film. Testing of drying time, water resistance, microcalorimetric analysis, compatibility studies, and in vitro release testing presented the possibility of evaluating the suitability of tested polymers for formulation of nail lacquers. The results provided reliable arguments for choosing Eudragit RL100 and Eudragit RS100 for further lacquer composition optimization studies. Optimization resulted in definition of quantitative composition of nail lacquers, containing both Eudragit RL100 and Eudragit RS100 as film-forming polymers, and suitability of designed formulations was confirmed by in vitro biopharmaceutical testing. The application of optimization technology proved to be efficient tool in designing and identifying naftifine hydrochloride lacquer compositions with predefined quality parameters. Quality of optimized nail lacquer formulations should be confirmed by performing transungual naftifine hydrochloride delivery studies. 


\section{Competing Interests}

The authors declare that there are no competing interests.

\section{Acknowledgments}

The authors are thankful to Lanxess, Evonik Industries AG, and Colorcon for generous supply of chemical substances.

\section{References}

[1] S. Naumann, J.-P. Meyer, A. Kiesow, Y. Mrestani, J. Wohlrab, and R. H. H. Neubert, "Controlled nail delivery of a novel lipophilic antifungal agent using various modern drug carrier systems as well as in vitro and ex vivo model systems," Journal of Controlled Release, vol. 180, no. 1, pp. 60-70, 2014.

[2] J. J. Finch and E. M. Warshaw, "Toenail onychomycosis: current and future treatment options," Dermatologic Therapy, vol. 20, no. 1, pp. 31-46, 2007.

[3] M. M. A. Elsayed, "Development of topical therapeutics for management of onychomycosis and other nail disorders: a pharmaceutical perspective," Journal of Controlled Release, vol. 199, pp. 132-144, 2015

[4] I. Vejnovic, L. Simmler, and G. Betz, "Investigation of different formulations for drug delivery through the nail plate," International Journal of Pharmaceutics, vol. 386, no. 1-2, pp. 185-194, 2010.

[5] H.-J. Tietz, R. Hay, S. Querner, A. Delcker, P. Kurka, and H. F. Merk, "Efficacy of 4weeks topical bifonazole treatment for onychomycosis after nail ablation with $40 \%$ urea: a double-blind, randomized, placebo-controlled multicenter study," Mycoses, vol. 56, no. 4, pp. 414-421, 2013.

[6] V. B. Rajendra, A. Baro, A. Kumari, D. L. Dhamecha, S. R. Lahoti, and S. D. Shelke, "Transungual drug delivery: an overview," Journal of Applied Pharmaceutical Science, vol. 2, no. 1, pp. 203-209, 2012.

[7] K. Frederiksen, R. H. Guy, and K. Petersson, "The potential of polymeric film-forming systems as sustained delivery platforms for topical drugs," Expert Opinion on Drug Delivery, vol. 13, no. 3, pp. 349-360, 2016.

[8] M. V. Saner, A. D. Kulkarni, and C. V. Pardeshi, "Insights into drug delivery across the nail plate barrier," Journal of Drug Targeting, vol. 22, no. 9, pp. 769-789, 2014.

[9] D. Monti, L. Saccomani, P. Chetoni, S. Burgalassi, M. F. Saettone, and F. Mailland, "In vitro transungual permeation of ciclopirox from a hydroxypropyl chitosan-based, water-soluble nail lacquer," Drug Development and Industrial Pharmacy, vol. 31, no. 1, pp. 11-17, 2005.

[10] D. J. Lunter and R. Daniels, "New film forming emulsions containing Eudragit ${ }^{\circledR} \mathrm{NE}$ and/or RS 30D for sustained dermal delivery of nonivamide," European Journal of Pharmaceutics and Biopharmaceutics, vol. 82, no. 2, pp. 291-298, 2012.

[11] L. A. Felton, "Mechanisms of polymeric film formation," International Journal of Pharmaceutics, vol. 457, no. 2, pp. 423-427, 2013.

[12] F. Hafeez, X. Hui, A. Chiang, S. Hornby, and H. Maibach, "Transungual delivery of ketoconazole using novel lacquer formulation," International Journal of Pharmaceutics, vol. 456, no. 2, pp. 357-361, 2013.

[13] D. Monti, L. Saccomani, P. Chetoni et al., "Hydrosoluble medicated nail lacquers: in vitro drug permeation and corresponding antimycotic activity," British Journal of Dermatology, vol. 162, no. 2, pp. 311-317, 2010.

[14] S. T. Tanriverdi and Ö. Özer, "Novel topical formulations of Terbinafine- $\mathrm{HCl}$ for treatment of onychomycosis," European Journal of Pharmaceutical Sciences, vol. 48, no. 4-5, pp. 628-636, 2013.

[15] K. R. Shireesh, S. B. Chandra, P. Vishnu, and M. V. V. Prasad, "Ungual drug delivery system of ketoconazole nail lacquer," International Journal of Applied Pharmaceutics, vol. 2, pp. 17-19, 2010.

[16] K. Frederiksen, R. H. Guy, and K. Petersson, "Formulation considerations in the design of topical, polymeric film-forming systems for sustained drug delivery to the skin," European Journal of Pharmaceutics and Biopharmaceutics, vol. 91, pp. 915, 2015.

[17] A. Subissi, D. Monti, G. Togni, and F. Mailland, "Ciclopirox: recent nonclinical and clinical data relevant to its use as a topical antimycotic agent," Drugs, vol. 70, no. 16, pp. 2133-2152, 2010.

[18] I. Z. Schroeder, P. Franke, U. F. Schaefer, and C.-M. Lehr, "Delivery of ethinylestradiol from film forming polymeric solutions across human epidermis in vitro and in vivo in pigs," Journal of Controlled Release, vol. 118, no. 2, pp. 196-203, 2007.

[19] C. Padula, S. Nicoli, P. Colombo, and P. Santi, "Single-layer transdermal film containing lidocaine: modulation of drug release," European Journal of Pharmaceutics and Biopharmaceutics, vol. 66, no. 3, pp. 422-428, 2007.

[20] K. V. Vipin, C. Sarath Chandran, A. R. Augusthy, K. Premaletha, and M. R. Kuriakose, "Formulation and evaluation of an antifungal nail lacquer for onychomycosis," British Biomedical Bulletin, vol. 2, no. 1, pp. 242-248, 2014.

[21] S. Murdan, L. Kerai, and B. Hossin, "To what extent do in vitro tests correctly predict the in vivo residence of nail lacquers on the nail plate?" Journal of Drug Delivery Science and Technology, vol. 25, pp. 23-28, 2015.

[22] F. Lecomte, J. Siepmann, M. Walther, R. J. MacRae, and R. Bodmeier, "Polymer blends used for the coating of multiparticulates: comparison of aqueous and organic coating techniques," Pharmaceutical Research, vol. 21, no. 5, pp. 882-890, 2004.

[23] P. Rama Rao and P. V. Diwan, "Permeability studies of cellulose acetate free films for transdermal use: influence of plasticizers," Pharmaceutica Acta Helvetiae, vol. 72, no. 1, pp. 47-51, 1997.

[24] A. S. Dessai and A. R. Shabaraya, "Formulation and in vitro characterization of nail lacquer containing fluconazole for preungual drug delivery system," International Journal of Pharmaceutical Research and Bio-Science, vol. 3, no. 2, pp. 200-214, 2014. 

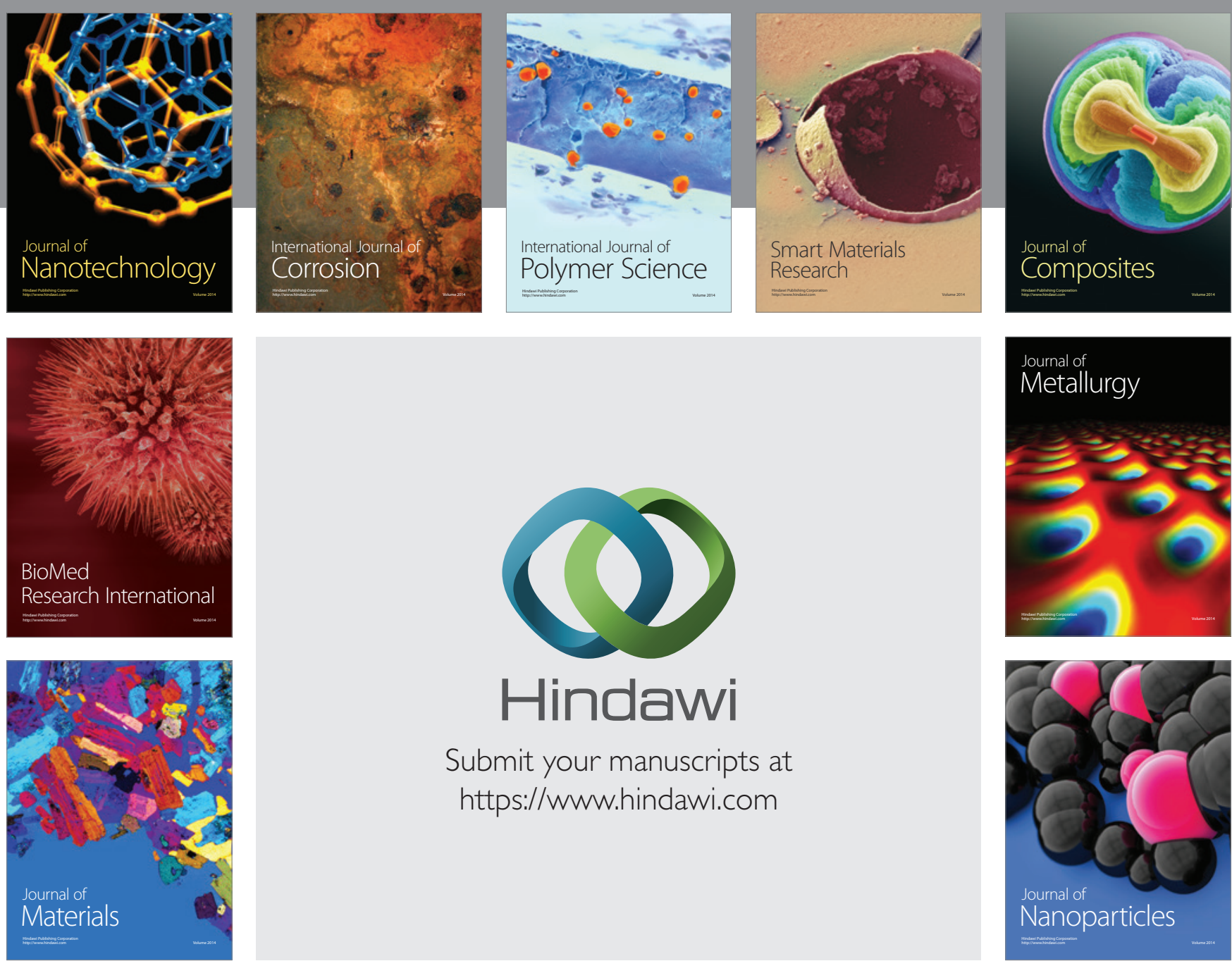

\section{Hindawi}

Submit your manuscripts at

https://www.hindawi.com

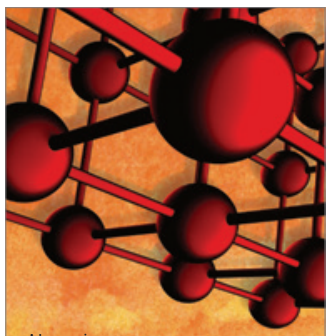

Materials Science and Engineering
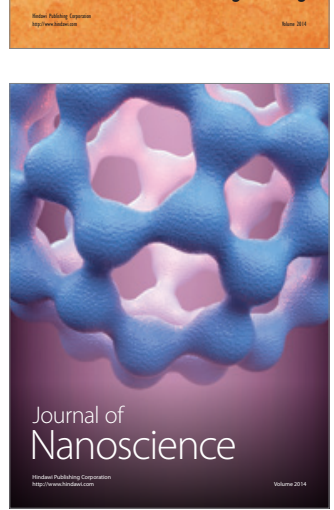
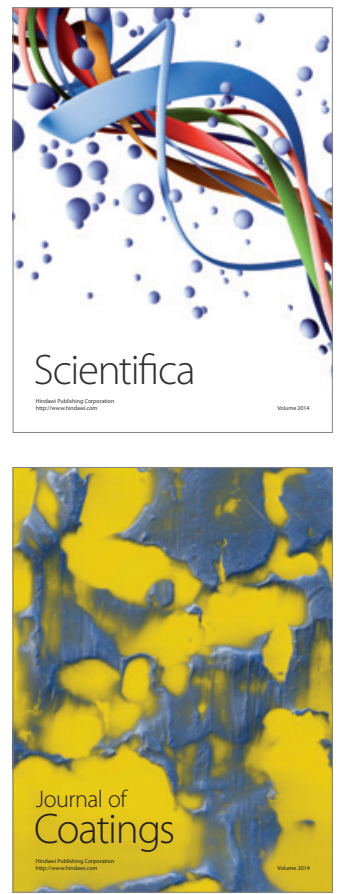
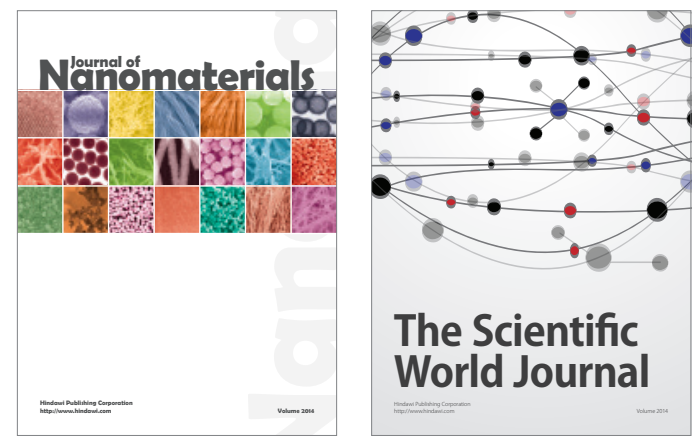

The Scientific World Journal
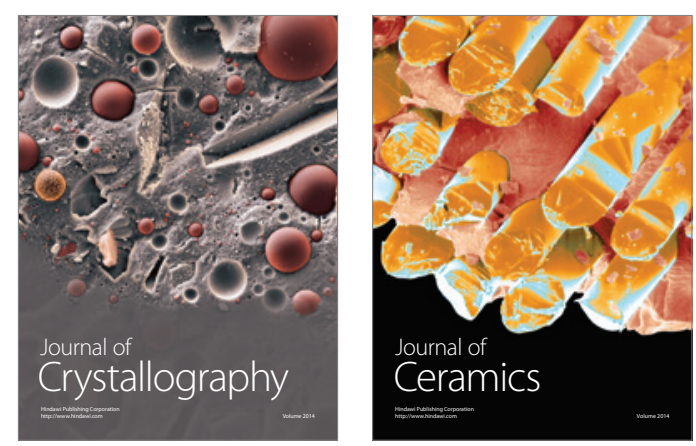
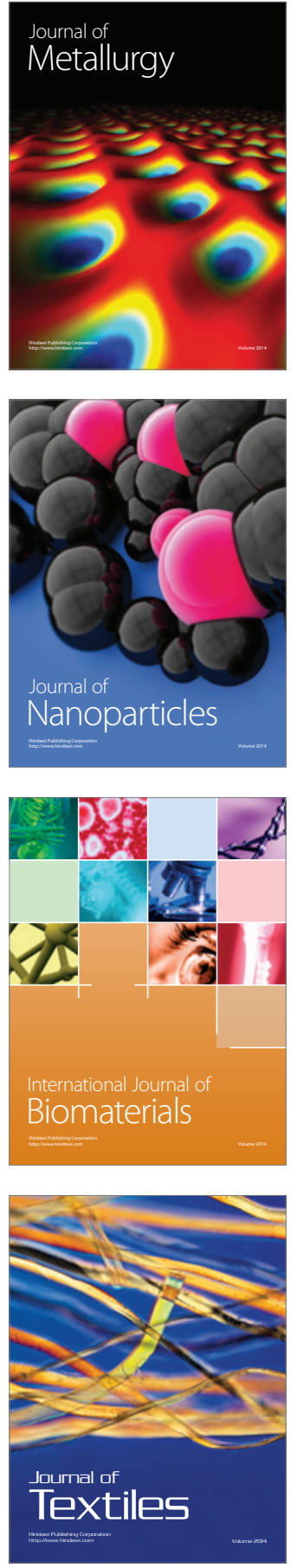